\title{
Hepatitis $C$ virus and lichen planus
}

\author{
Is there an association between hepatitis $\mathrm{C}$ and oral lichen planus?
}

\section{Chainani-Wu N, Lozada-Nur F, Terrault N. Hepatitis $C$ virus and lichen planus: a review. Oral Surg Oral Med Oral Pathol Oral Radiol Endod 2004; 98:171-183}

Data sources Medline (1966-June 2003) provided the primary data source, with references from identified papers then being reviewed for additional studies.

Study selection Studies were included if they reported prevalence of hepatitis $C$ virus (HCV) in people who have oral lichen planus (OLP), compared clinical and histological features of OLP in patients who were or were not infected with HCV, reported presence of HVC within OLP lesions; or were studies of HCV genotypes in patients who had OLP lesions.

Data extraction and synthesis The available studies varied widely in design, population and distribution of confounders so could not be combined in any meaningful meta-analysis. Data were, therefore, summarised and tabulated.

Results In total, 32 studies were identified, of which 14 were descriptive, reporting prevalence, and 18 were case-control. HCV prevalence varied from 0 to $62 \%$ in individuals who had OLP. OLP prevalence varied from 1.6 to $20 \%$ in HCV-infected subjects. Important biases, including selection and investigator bias were identified within the studies.

Conclusions No firm conclusions can be drawn from this review because of the diverse nature of the available studies and the biases contained therein.

\section{Commentary}

Lichen planus is a relatively common condition, thought to be a mucocutaneous, cell-mediated reaction, which can be precipitated by a number of factors including microbiological agents. OLP has been described in association with numerous systemic diseases, particularly those affecting the liver. Thus, when Mokni et al. ${ }^{1}$ (1991) described a patient with both OLP and hepatitis C virus (HCV) infection, a possible association between the two conditions was investigated by a large number of studies, often with conflicting results.

The aim of the paper by Chainani-Wu and co-workers is, they state, to review and summarise the published literature on the association between OLP and HCV, "and to describe future directions". Thanks to its clear style the article is comprehensible even by clinicians who do not have a strong methodological and statistical background. The authors base their narrative review on the citations resulting from a simple, but probably very sensitive, research strategy. This was performed using Medline alone but missed few relevant papers.

The major merit of the review is that it extensively examines every aspect of the problem. Articles are sorted on the basis of aims and design and are briefly described, summarised in tables and discussed, giving the reader a wide overview of most of the

Address for correspondence: Nita Chainani-Wu, Department of Stomatology, Division of Oral Medicine, Oral Pathology and Oral Radiology, University of California, San Francisco CA 94143-0658, USA. E-mail: nitacwu@itsa.ucsf.edu published information investigating (a) prevalence of HCV infection in OLP patients; (b) prevalence and incidence of OLP in HCVinfected individuals; and (c) pathological mechanisms explaining the association of the two conditions.

This inclusive approach to the literature also limits the review, since it was not designed to provide evidence-based conclusions to the reader. The lack of explicit inclusion criteria results in a group of studies that is too heterogeneous, as admitted by authors, to make possible a quantitative analysis of the results. Also, the criteria used to assign the included studies to categories (case report, descriptive studies, case-control studies) are not stated, causing some confusion to the reader. A case-control study (reference 18 in the review) is included in "Descriptive studies", whereas studies comparing HCV prevalence of an OLP group with historical data from the general population are included either in 'Descriptive studies' (in the case of review references 14 and 17) or in 'Case-control studies' (for review reference 26). A study that never mentions oral involvement (reference 34 ) is included in the OLP-studies category. The problem may result from the fact that the authors were not able to obtain full text of all the articles, and based their assessment on abstracts only.

A critical appraisal of the studies is attempted: a number of possible confounding factors and bias able to affect the results and explain between-study variability are described. In most cases, these are not assessed in the single studies, however, because of reporting problems (many papers lack of relevant information) or lack of information (unavailability of full text in some cases). No clear quality criteria were established for the different study designs, also, making it difficult (impossible?) for the reader to decide which study can be trusted (internal validity) and its results applied in practice (external validity).

In conclusion, this paper is a narrative review that addresses a broad question (to investigate the epidemiological and pathological aspect of the OLP-HCV association). It may be useful to the reader as a summary of the literature, but cannot provide any evidencebased answer.

\section{Practice point}

- This review usefully summarises aspects of an OLP-HCV association but there is no evidence to answer to question.

\section{Giovanni Lodi}

Unità di Medicina e Patologia Orale, Dipartimento di Medicina Chirurgia e Odontoiatria, Università degli Studi di Milano, via Beldiletto 1/3 Milano 20142, Italy

1. Mokni M, Rybojad M, Puppin Jr D, et al. Lichen planus and hepatitis C virus. J Am Acad Dermatol 1991; 24:792.

Evidence-Based Dentistry (2006) 7, 18.

doi:10.1038/sj.ebd.6400379 